\title{
Using Interface Refinement to Integrate Formal Verification into the Design Cycle*
}

\author{
Jacob Chang, Sergey Berezin, and David L. Dill \\ Stanford University \\ \{Jacob.Chang, berezin, dill\}@stanf ord.edu
}

\begin{abstract}
We present a practical compositional interface refinement methodology which helps to integrate formal verification with the design process. One of the main verification challenges is to keep up with the changes to the specifications as the design evolves, and in particular, the transformations to the interfaces between the components. Interface changes are usually incremental, and therefore, the verification efforts after each change should also be incremental in order to be scalable. This paper presents a compositional interface refinement methodology which addresses all of these issues.

We have applied this methodology in the design process of a network controller that will be used in the Smart Memory project at Stanford. The design is split into modules which are modeled as transition systems, and the properties of their interfaces are described in the First-Order LTL. We perform interface refinement by compositional reasoning with the help of an additional interface converter module. Unlike in previous approaches, the inverse converter does not need to be constructed, thus, making the verification of the refinement step simpler.
\end{abstract}

\section{Introduction}

The advantage of formal verification over simulation is that one can verify all possible behaviors of a design. This is especially important if the design is complex and has many corner cases that might not be covered by simulation. However, automated methods of formal verification have been limited to relatively small designs $[15,5,8,1]$. In order to verify a large design formally, one can apply compositional reasoning and refinement techniques [6,9] to split the verification of the low-level implementation into manageable parts. Compositional refinement techniques guarantee that such decomposition preserves the correctness of the entire design.

In this paper, we describe a new compositional interface refinement technique, which is similar to the traditional compositional refinement techniques, except that when an abstract component is refined to its concrete version, its interface is also allowed to change.

\footnotetext{
* This research was supported in part by DARPA contract F29601-032-0117 and NSF grant CCR 0121403.
} 


\subsection{Interface Refinement}

It is a long held belief that if one could integrate formal verification into the design process, then it would make formal verification easier to perform, and improve the design process. Such tight integration was the key to successful microprocessor verification by Kroening in [11].

Integration of formal verification into a top-down design methodology means that a design starts at an abstract level for which exhaustive verification is done. Then, as the design evolves to a more detailed implementation, compositional techniques can be used in order to verify the more detailed design.

However, most of the existing compositional refinement approaches require that the interfaces among the components do not change in the refinement process. This is not an easy requirement to meet, because engineering specifications become more detailed as the design evolves, and this necessitates the refinement of the interfaces. In order to integrate formal verification into the design process, the verification methodology must allow for interface changes that will occur during the course of the design. The problem of verifying that the changes to the interfaces preserve the correctness of the design is called the interface refinement problem.

In this paper, we present a methodology that addresses the interface refinement problem in the context of compositional verification. This allows the verification effort to follow the interface changes during the design and avoids the unnecessary restriction on the interfaces imposed by the traditional refinement techniques.

\subsection{Our Approach}

The problem of interface refinement has been recognized in the past, and a few approaches provide formalization of this problem $[3,18,4,7,12]$. These results have built several theoretical foundations for interface refinement, but their practical application in real engineering projects so far have been limited.

The most practical approach to interface refinement, to the best of our knowledge, is the method presented by Brinksma et al [3]. Their approach is based on a process algebra framework, and the interfaces are refined through the use of a converter module that converts between the abstract and the concrete interfaces. One of the requirements of this methodology is that the converter must have an inverse.

From our experience with the verification of the Smart Memory network controller, we found that the specifications are more naturally expressed in LTL rather than in process algebra. It is also natural to define the converter as a circuit which translates from the abstract interface to the concrete interface. Furthermore, the inverse of the converter may not exist.

In section 2 we present a generic interface refinement methodology. In particular, it works with the LTL specifications and does not require the existence of the inverse converter. We illustrate our interface refinement methodology by a simple example, then discuss its practical application to the Smart Memory network controller in section 3 , and section 4 concludes the paper. 


\section{Compositional Interface Refinement}

Compositional refinement has been extensively studied in the past [6,14], and is now a standard approach to verification of complex systems. We briefly remind the basics of this approach and introduce the formal notation.

For simplicity, let us consider a design consisting of just two modules, $X$ and $Y$, connected together in parallel by the parallel composition operator: $X \| Y$, which we deliberately leave undefined. This operator is assumed to be associative: $(X \| Y)\|Z=X\|(Y \| Z)$, so a composition of more than two modules can be written simply as $X\|Y\| Z$.

Provided that our design $X \| Y$ satisfies the property $\phi$ (denoted $X \| Y \models$ $\phi$ ), our goal is to replace each component with a more detailed (or refined) implementations $X^{\prime}$ and $Y^{\prime}$, respectively, and show that the resulting system $X^{\prime} \| Y^{\prime}$ also satisfies $\phi$.

More formally, we introduce a refinement relation $\preceq$ over modules which preserves the correctness properties from a certain class (e.g. properties expressible in LTL). That is, if $X \models \psi$ and $X^{\prime} \preceq X$, then $X^{\prime} \models \psi$ for any property $\psi$ from the chosen class. The refinement relation must be transitive: $X^{\prime \prime} \preceq X^{\prime}$ and $X^{\prime} \preceq X$ imply $X^{\prime \prime} \preceq X$. In addition, we require that the parallel composition operator is associative and monotonic w.r.t. $\preceq$ for both arguments. That is, if $X^{\prime} \preceq X$, then $X^{\prime}\|Y \preceq X\| Y$ and $Y\left\|X^{\prime} \preceq Y\right\| X$.

For this section, we leave the operators $\|$ and $\preceq$ undefined, and only assume the following algebraic properties: associativity of $\|$, transitivity of $\preceq$, and monotonicity of $\|$ w.r.t. $\preceq$. This allows the results to be used in various contexts. In particular, we define these operators in a somewhat unusual fashion, over LTL formulas, in section 2.2 .

Since the refined components $X^{\prime}$ and $Y^{\prime}$ may be rather complex, in order to simplify the verification problem, we would like to reason about each component individually, and avoid verifying $X^{\prime} \| Y^{\prime}$ directly. A well-known approach to this problem is know as compositional refinement [9], which essentially boils down to showing that

$$
X^{\prime} \preceq X \quad \text { and } \quad Y^{\prime} \preceq Y .
$$

By the monotonicity of $\|$ and transitivity of $\preceq$ the properties (1) imply $X^{\prime} \| Y^{\prime} \preceq$ $X \| Y$, and hence, $X^{\prime} \| Y^{\prime} \models \phi$, provided that $X \| Y=\phi$.

Notice that we can show (1) only if modules $X^{\prime}$ and $Y^{\prime}$ have the same interfaces as $X$ and $Y$, respectively. However, in real designs, the interfaces often change as the specifications get refined, as described in section 1 , and it is often desirable to refine the interfaces together with the modules.

The problem of interface refinement consists in verifying the refinement of a composite module $X\left\|Y \preceq X^{\prime}\right\| Y^{\prime}$ when the communication interface between the two components changes. For example, we have module $X$ and $Y$ communicating through an abstract interface $c$ as shown in the top portion of figure 1. We want to refine $X$ and $Y$ to $X^{\prime}$ and $Y^{\prime}$ such that they communicate over a different protocol $d$, but their composition $X^{\prime} \| Y^{\prime}$ should still have a combined property $\phi$, given that the abstract system $X \| Y$ satisfies the same property $\phi$. 
Notice that if we do indeed change the protocol, the direct compositional refinement does not go through, since the refinement of the individual modules (1) fails.

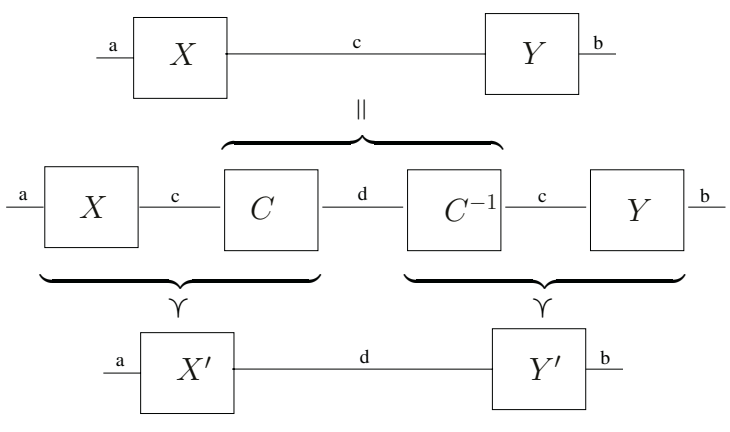

Fig. 1. Interface refinement by Brinksma et al

The interface refinement technique suggested by Brinksma et al [3] (translated to our notation) is to show the following refinements:

$$
X^{\prime} \preceq X \| C \quad \text { and } \quad Y^{\prime} \preceq C^{-1} \| Y
$$

where $C$ and $C^{-1}$ are protocol converters (figure 1 ). From the monotonicity of $\|$, it immediately follows that $X^{\prime}\left\|Y^{\prime} \preceq X\right\| C\left\|C^{-1}\right\| Y$. Since $C^{-1}$ is the inverse of $C$, the composition of the two converters $C^{-1} \| C$ is exactly equivalent to the identity function (that is, any input to $C$ appears as the output of $C^{-1}$ ). This implies that $X\|C\| C^{-1}\|Y=X\| Y$, and hence, $X^{\prime}\left\|Y^{\prime} \preceq X\right\| Y$, which is the desired result of the refinement verification.

The problem with this approach is that it is often difficult to find the inverse $C^{-1}$ of the converter $C$. For instance, if the interface changes from a wide parallel bus (abstract interface) that sends data in a single clock cycle to a serial bus (concrete interface) sending the same data over several clock cycles, the serialto-parallel converter $C$ would have to "predict the future" in order to output the data the moment it enters the inverse converter $C^{-1}$. One way to get around this problem is to use the concept of stuttering equivalence [13]. However, we have found that showing that the composition of the converters is the identity model is also difficult under this model.

\subsection{Compositional Interface Refinement: Our Approach}

Since the abstract interface and the converters will not appear in the concrete implementation, we would like to take advantage of this flexibility in our refinement proof. Thus, we propose the following method for interface refinement. 
First, we only perform the refinement on one of the modules, say module $Y$, by showing that $C \| Y^{\prime} \preceq Y$, where $C$ is a converter module translating the abstract protocol $c$ to the concrete protocol $d$. By compositional reasoning, $X\|C\| Y^{\prime} \preceq X \| Y$ (figure 2), and thus, $X\|C\| Y^{\prime}$ also satisfies property $\varphi$. Next, instead of performing the same refinement step on module $X$, we show that the concrete module $X^{\prime}$ is a refinement of the composition $X \| C$. Putting the two refinements together completes the refinement chain: $X^{\prime}\left\|Y^{\prime} \preceq X\right\| C\left\|Y^{\prime} \preceq X\right\| Y$.

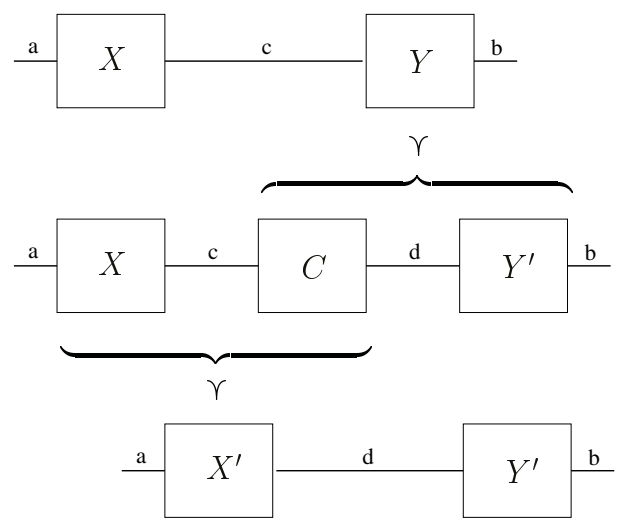

Fig. 2. Interface refinement: our method

Note, that our approach requires only one interface converter, in the direction of our choice; the inverse converter is not needed. This method is also quite general, since it relies on the same algebraic properties of the composition $\|$ and refinement $\preceq$ as the traditional compositional reasoning, and therefore, can be applied in any framework where such properties are satisfied. In particular, the method is applicable even when the composition and refinement operators are defined in non-standard ways (one of which is described in the next section), as long as the required algebraic properties are satisfied.

\subsection{First-Order LTL}

In the practical application of our compositional interface refinement method, we use a version of First-Order Linear-time Temporal Logic (LTL) similar to the one used in the STeP tool [2] at Stanford. LTL properties are preserved by trace inclusion, the type of refinement relation where the refined component has fewer behaviors, or traces than the abstract component. This logic is expressive enough to permit compositional reasoning with interface refinement directly in the logic. Additionally, LTL is the specification language of Cadence SMV[16], the primary tool we have used to perform the actual verification. 
Syntax. We assume two disjoint sets of logical and state variables, $V_{L}$ and $V_{S}$. The syntax of an LTL formula $\phi$ is given by the following BNF form:

$$
\phi::=p\left|\phi_{1} \wedge \phi_{2}\right| \neg \phi_{1}\left|\mathbf{G} \phi_{1}\right| \mathbf{F} \phi_{1}\left|\mathbf{X} \phi_{2}\right| \phi_{1} \mathbf{U} \phi_{2}\left|\exists^{s} u . \phi_{1}\right| \forall^{s} u . \phi_{1}
$$

where $p$ is an atomic formula, $\phi_{1}$ and $\phi_{2}$ are formulas and $u \in V_{S}$ is a state variable. Atomic formulas are predicates over state variables.

Semantics. The semantics of an LTL formula $\phi$ is defined over all paths in a model (Kripke structure) $M=\left(S, S_{0}, \rightarrow, D\right)$, where $S$ is the set of states, $S_{0} \subseteq S$ is the set of initial states, $\rightarrow \subseteq S \times S$ is the transition relation, and $D$ is the domain of state and logical variables. A state $s \in S$ is an assignment of values from $D$ to state variables; that is $s: V_{S} \rightarrow D$. We also introduce a logical variable assignment $e: V_{L} \rightarrow D$.

The semantics of a LTL formula $\phi$ is defined over a sequences of states called paths $\pi$ that represent execution traces of the model. A state is an assignment to variables that occur in $\phi$ and, possibly, to other variables. The temporal operators are the same as in propositional LTL. For example, $\pi \models \mathbf{G} \phi$ means that $\phi$ is true everywhere along the path. A quantified formula $\pi \models \forall^{s} u$. $\phi$ holds if $\phi$ holds for all paths $\pi^{\prime}$ such that $\pi^{\prime}$ is the same as $\pi$ except for assignment to the state variable $u$. An existential formula $\pi \models \exists^{s} u$. $\phi$ is defined similarly except that we only need to find one such $\pi^{\prime}$ which satisfies $\phi$.

An LTL formula $\phi$ holds in a model $M$ (denoted $M \models \phi$ ) whenever it holds on any path starting from an initial state of $M$. We say that a formula $\phi$ is valid $(\mid=\phi)$ whenever it holds in any model.

Compositional Refinement in LTL. The refinement property in this framework is defined as trace inclusion, where trace is an execution path $\pi$ of a particular module $A$ starting from some initial state of $A$. Recall that in the previous section, we left $\|$ and $\preceq$ undefined and only require that they satisfy certain algebraic properties. In this section, we define these operator in a non-standard way: over LTL formulas. If two modules $A$ and $A^{\prime}$ satisfy LTL properties $\phi_{A}$ and $\phi_{A^{\prime}}$ respectively, then $A^{\prime} \preceq A$ is defined by the following formula:

$$
\phi_{A^{\prime}} \rightarrow \exists^{s} \boldsymbol{u} \cdot \phi_{A}
$$

where $\boldsymbol{u}$ is the vector of all the state variables of $\phi_{A}$ which do not occur in $\phi_{A^{\prime}}$.

Parallel composition of two modules $A \| B$ is simply a conjunction of their respective properties: $\phi_{A} \wedge \phi_{B}$. The fact that a composition of modules satisfies some property $\phi$ can now be derived by

$$
\phi_{A} \wedge \phi_{B} \rightarrow \phi
$$

These definitions of $\|$ and $\preceq$ clearly satisfy the required algebraic properties stated in section 2.1. Effectively, the actual modules are replaced by their LTL formulas, and all the compositional reasoning is now done completely in LTL. 
Interface Refinement in LTL. Our approach to compositional interface refinement translates to the LTL framework as follows. Suppose that the properties $X \models \phi_{X}$ and $Y \models \phi_{Y}$ have already been verified, and the desired property of their composition $X \| Y \models \phi$ follows from the fact that

$$
\models \phi_{X} \wedge \phi_{Y} \rightarrow \phi .
$$

Additionally, we assume that the concrete modules satisfy their respective properties:

$$
X^{\prime} \models \phi_{X^{\prime}} \quad Y^{\prime} \models \phi_{Y^{\prime}} .
$$

Recall in section 2.1, in order to show that the concrete design also satisfies $\phi$, that is, $X^{\prime} \| Y^{\prime} \models \phi$, it is sufficient to show that $C \| Y^{\prime} \preceq Y$ and $X^{\prime} \preceq X \| C$, which translates to LTL as follows:

$$
\begin{aligned}
\phi_{C} \wedge \phi_{Y^{\prime}} & \rightarrow \phi_{Y} \\
\phi_{X^{\prime}} & \rightarrow \exists^{s} c . \phi_{X} \wedge \phi_{C}
\end{aligned}
$$

where $\phi_{C}$ is some formula representing the converter module $C$. Equation (6) does not have the existential quantifier because all the free variables in $\phi_{Y}$ appear on the left hand side of the implication. Note, that there are no restrictions on the formula $\phi_{C}$. In particular, the actual converter $C$ does not have to be built (and may, in fact, be unimplementable), and $C \models \phi_{C}$ does not need to be checked.

\section{Examples and Applications}

In this section, we first demonstrate the practical application of our method on a very simple example: composition of two modules passing an $L$-bit value from input to output over an $L$-bit wide bus, and its refinement to the two modules which communicate through a serial bus (1-bit wide). Then we describe a more realistic example of an actual design in the context of the Smart Memory Project.

\subsection{A Simple Example for Illustration}

Suppose that we have two modules $X$ and $Y$ that pass the input from $a$ to $c$ and from $c$ to $b$ as shown in figure (3a), modifying the data with functions $f_{X}$ and $f_{Y}$; that is, given the input $D$, the corresponding output will be $f_{Y}\left(f_{X}(D)\right)$. Signal $c$ is a parallel interface that is $L$ bits wide, and is represented as a record

$$
c:\{\text { valid : bool, data : bool }[L]\} \text {. }
$$

The transmission of data occurs when the valid bit is set to 1 , and the data field is supposed to contain the valid data in the same cycle. The property that the 


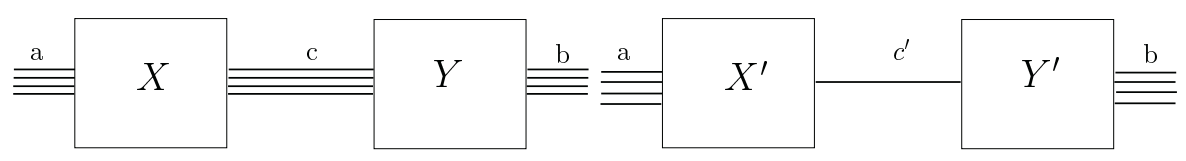

(a) parallel interface

(b) serial interface

Fig. 3. Systems with parallel and serial interfaces

data $D: \operatorname{bool}[L]$ is sent over the channel $c$ at the current cycle is expressed in LTL as follows:

$$
c \rightrightarrows D \triangleq[c . \text { alid }=1 \wedge c . \text { data }=D]
$$

The property of the composed system is then written as follows:

$$
\phi \triangleq \forall(D: \operatorname{bool}[L]) . \mathbf{G}\left(a \rightrightarrows D \rightarrow \mathbf{F}\left[b \rightrightarrows f_{Y}\left(f_{X}(D)\right)\right]\right) \text {. }
$$

Suppose that the abstract components $X$ and $Y$ satisfy the properties $\phi_{X}$ and $\phi_{Y}$, respectively, where

$$
\begin{aligned}
& \phi_{X} \triangleq \forall D . \mathbf{G}\left(a \rightrightarrows D \rightarrow \mathbf{F}\left[c \rightrightarrows f_{X}(D)\right]\right) \\
& \phi_{Y} \triangleq \forall D . \mathbf{G}\left(c \rightrightarrows D \rightarrow \mathbf{F}\left[b \rightrightarrows f_{Y}(D)\right]\right),
\end{aligned}
$$

and we have already established that

$$
\phi_{X} \wedge \phi_{Y} \rightarrow \phi
$$

The concrete implementation of the system consists of two components $X^{\prime}$ and $Y^{\prime}$ which communicate over a serial bus $c^{\prime}$ as shown in figure (3b).

A serial interface $c^{\prime}$ is a record, similar to the concrete interface, only the data field is one bit wide:

$$
c^{\prime}:\{\text { first_bit : bool, bit : bool }\}
$$

A valid transmission of an $L$-bit data $D$ over the serial bus $c^{\prime}$ starts when the first_bit field becomes 1 ; at this point, the bit field contain the first bit of the data, $D[0]$, and in the subsequent $L-1$ cycles first_bit remains 0 while the bit field takes the values of the remaining bits $D[1]$ through $D[L-1]$. Such a transmission is expressed in LTL as the following formula:

$$
\begin{aligned}
c^{\prime} \rightsquigarrow D \triangleq & c^{\prime} \text {.first_bit }=1 \wedge \text { bit }=D[0] \\
& \wedge \bigwedge_{i=1}^{L-1} \overline{\mathbf{X}}^{i}\left(c^{\prime} \text {.first_bit }=0 \wedge c^{\prime} \text {.bit }=D[i]\right),
\end{aligned}
$$


where $\mathbf{X}^{i} \phi$ is an abbreviation for $\underbrace{\mathbf{X} \ldots \mathbf{X}}_{i} \phi$. The properties of the concrete modules can now be written as follows:

$$
\begin{aligned}
& \phi_{X^{\prime}} \triangleq \forall D \cdot \mathbf{G}\left(a \rightrightarrows D \rightarrow \mathbf{F}\left[c^{\prime} \rightsquigarrow f_{X}(D) \wedge \bigwedge_{i=0}^{L-1} \mathbf{X}^{i} X^{\prime} \cdot d=f_{X}(D)\right]\right) \\
& \phi_{Y^{\prime}} \triangleq \forall D \cdot \mathbf{G}\left(c^{\prime} \rightsquigarrow D \rightarrow \mathbf{F}\left[b \rightrightarrows f_{Y}(D)\right]\right) .
\end{aligned}
$$

Here $X^{\prime} . d$ is the internal buffer of $X^{\prime}$ holding the data currently being transmitted on its output bus $c^{\prime}$. The formula $\phi_{X^{\prime}}$ states that $X^{\prime}$ will eventually start sending the data it receives on the input $a$, and while the transmission is active, the buffer $X^{\prime} . d$ will hold the entire data being transmitted. In this case, we would like to establish that

$$
\phi_{X^{\prime}} \wedge \phi_{Y^{\prime}} \rightarrow \phi \text {. }
$$

However, we do not want to prove this formula directly, and instead, use the already proved property (8). As the first step, we come up with the following property:

$$
\phi_{C} \triangleq \forall D . \mathbf{G}\left(c \rightrightarrows D \rightarrow c^{\prime} \rightsquigarrow D\right)
$$

which a protocol converter $C$ would satisfy if we were to build it. Note, that the converter has no delay between receiving the parallel data and starting to send the same data over the serial output. As such, this module is unimplementable, since the parallel input may exceed the data rate of the serial output. However, this property is sufficient to prove the correctness of the refinement.

According to our methodology, in order to establish (9), it is sufficient to show that the following two formulas hold:

$$
\begin{gathered}
\phi_{C} \wedge \phi_{Y^{\prime}} \rightarrow \phi_{Y} \\
\phi_{X^{\prime}} \rightarrow \exists c . \phi_{X} \wedge \phi_{C} .
\end{gathered}
$$

These two refinements are illustrated in figures 4(a) and 4(b). In (11), the parallel composition $X \| C$ has a nondeterministic state variable $c$, which is the state of the internal wire between $X$ and $C$. In order to show that $X^{\prime}$ is a refinement of $X \| C$, we need to show that for any trace of $X^{\prime}$ there exists an equivalent trace of $X \| C$. A particular trace of $X \| C$ can be chosen by a particular sequence of values for the variable $c$. This fact is denoted by the existential quantifier over $c$. When we provide an instantiation for $c$ as

$$
c \triangleq\left\{\text { valid }=c^{\prime} \text {.first_bit, data }=X^{\prime} . d\right\},
$$

then it will yield the equivalent trace of $X \| C$ for any trace of $X^{\prime}$. Note, that this instantiation also prevent overflowing the converter $C$ with excessive data on the input, since it can only receive the data as fast as $X^{\prime}$ can send it, which is exactly the rate of the serial bus. From this point, proving (10) and (11) is a relatively simple exercise. 


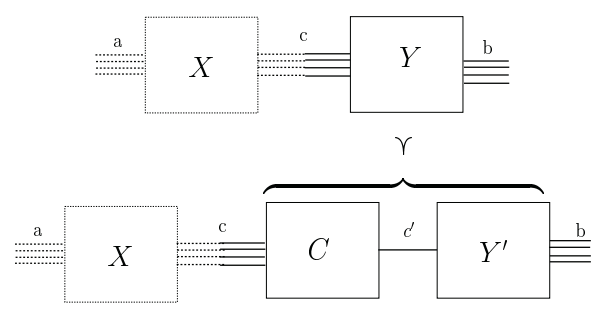

(a)

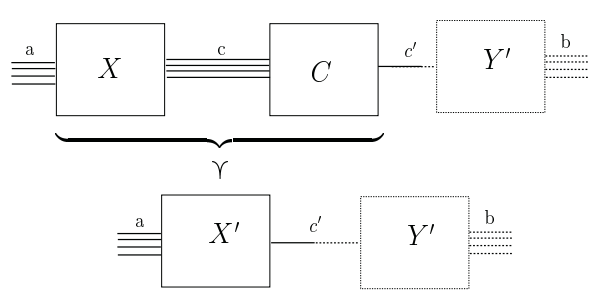

(b)

Fig. 4. Refinement of $\mathrm{X}$ and $\mathrm{Y}$.

\subsection{Application to Smart Memory Network Controller}

The Smart Memory project aims at developing a single chip multi processor system with reconfiguration capabilities to support various computing models. An on-chip network is required in this project to allow different sets of processors on the chip to communicate with each other.

When the design of the Smart Memory network controller was started, it was clear that getting it right would be a quite difficult task due to the complex interactions among multiple nodes in the network. Therefore, we decided to use formal verification in the design process to guarantee the correctness from the early stages, and preserve it as the design evolves. The practical demands of this engineering effort required the use of effective compositional reasoning together with interface refinement techniques, which eventually led to the results presented in this paper.

Smart Memory Network description. The Smart Memory chip is divided up into 16 nodes. The Smart Memory network passes short control messages as well as large chunks of data between these nodes. The topology of the network is such that each node connects to its four neighbors as well as the nodes that are two spaces away. Thus each quad has six input and output ports as shown in figure 5. Since the messages are of different sizes, we split the messages into smaller fixed length data unit called flits which are then transmitted over the network. To meet the latency constraints, all the routing information is placed in the header flit, and the data flits follow the same route. The route is determined dynamically by allocating physical connections, which constitute a limited shared resource. Since messages are sent in parallel, it is possible for the system to deadlock when two streams of flits are waiting for each other's resources.

Problem Statement. To formally verify the correctness of the network, we need to prove the guaranteed delivery of messages to the correct destination 


\section{Network Topology}

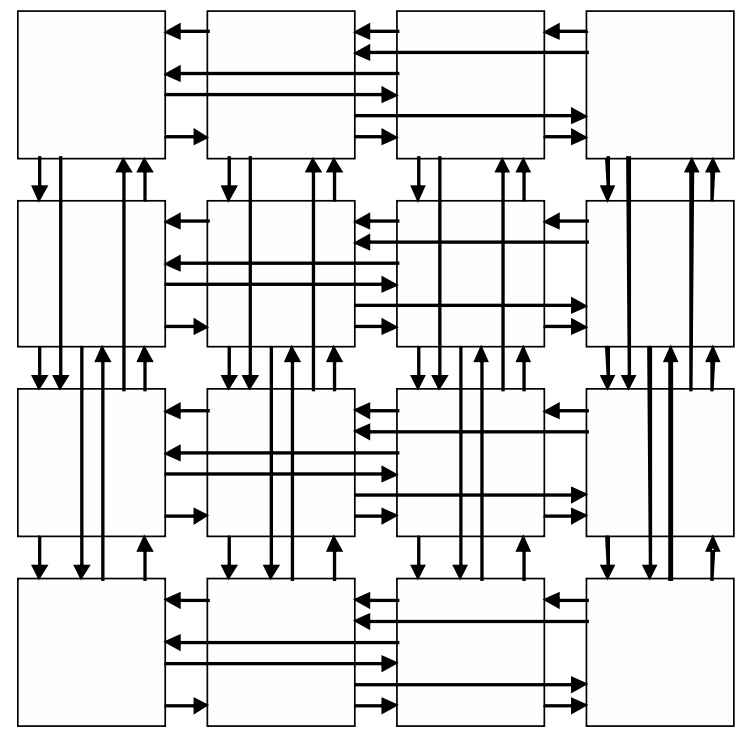

Fig. 5.

without duplication or loss. Proving that the routing algorithm correctly routes messages is already an interesting verification problem. The fact that messages are not atomic but consist of individual flits makes the verification problem even more difficult.

High level description of the interface refinement process. To simplify the verification process, we generalize the network to have arbitrary number of nodes and a parameterized connection scheme with certain restrictions on the topology (e.g. each input port is connected an output port, there is a route between every two nodes, etc.). At the abstract level, we describe the messages delivered by the network in terms of packets. The specification of each node acting on packets are specified by their properties in the assume-guarantee style. These restriction and specifications are easily encoded in LTL. We prove the correctness of packet routing given the node specifications without having to worry about the complexity of breaking up the packet into flits.

Then we refine the interface such that the communication between nodes are flits instead of packets. The correctness of the refined interface are checked by using our tool to process the LTL specifications and converted into verification conditions (7) and (10) which are then proved by Cadence SMV. The problem of refining the message interface to the flit interface is similar in spirit as the example illustrated in section 3.1. However, the fact that each node is connected 
to many other nodes, the message length can be of different size, and the presence of a flow control mechanism make the problem much more complex.

We then perform another refinement of the interface by adding the flow control to the flit interface using the same methodology.

We estimate that the the first refinement step took us about 10 hours, and about 10 hours were spent on expanding the existing toolset, although this does not include the time spent in our preliminary work with a similar simpler example to develop this methodology. The verification run performed by SMV for each verification condition our tool generate all finish within a minute.

\section{Conclusion}

We have demonstrated that interface refinement allows us to reuse the correctness proof of a design as the designer makes changes to the interface or makes the interface more concrete during the design cycle. Interface refinement also allows us to apply the divide-and-conquer technique to a large design by incrementally making the protocol more complex.

By applying our technique to the Smart Memory network controller during the design process, we have demonstrated that our interface refinement methodology can be used to allow formal verification to be done in parallel with design. Currently, the Smart Memory Network controller is still in the design stage and no concrete design have been written, therefore we have not made the link between the Hardware Description Language (HDL) description of the design with the LTL specification we have used for the modules. However, SMV is known for proving the LTL specification of the HDL description [10,17], and we foresee that it is possible to verify the HDL description of the network controller.

In addition to the method presented in this paper, we developed additional techniques specific to our toolset to perform the interface refinement of the network controller presented in this paper. These techniques allow us to describe the specifications in a more intuitive manner and deal with the more complex configuration such as a circular configuration.

The interface refinement methodology is being developed as a part of the larger project to formally verify the Smart Memory network controller, showing that formal verification can be applied to a large engineering design. In the future, we hope to demonstrate the feasibility of formally verifying a large design project by integrating verification into the design process. We believe that developing a methodology with the feedback and the pressure of the design team will allow us to address the important issues that are hindering formal verification from being commonly used.

Acknowledgements. The authors would like to thank Mark Horowitz and the Smart Memory design team for guiding the direction of this research. We would also like to thank the anonymous reviewers for helpful comments on the paper. 


\section{References}

1. C. Barrett, D. L. Dill, and J. Levitt. Validity checking for combinations of theories with equality. In Mandayam Srivas and Albert Camilleri, editors, Formal Methods In Computer-Aided Design, volume 1166 of Lecture Notes in Computer Science, pages 187-201. Springer-Verlag, November 1996.

2. N. Bjørner, A. Browne, M. Colon, B. Finkbeiner, Z. Manna, H. Sipma, and T. Uribe. Verifying temporal properties of reactive systems: A STeP tutorial. Formal Methods in System Design, 1999.

3. E. Brinksma, B. Jonsson, and F. Orava. Refining interfaces of communicating systems. In S. Abramsky and T. S. E. Maibaum, editors, TAPSOFT '91, Volume 2, volume 494 of $L N C S$, pages 297-312. SV, 1991.

4. M. Broy. Interaction refinement-the easy way. In Program Design Calculi: Proceedings of the 1992 Marktoberdorf International Summer School. Springer-Verlag, 1993.

5. J. R. Burch and D. L. Dill. Automatic verification of pipelined microprocessor control. In D. L. Dill, editor, Computer Aided Verification (CAV'94), volume 818 of LNCS. Springer-Verlag, 1994.

6. E. M. Clarke, D. E. Long, and K. L. McMillan. Compositional model checking. In Fourth Annual Symposium on Logic in Computer Science, pages 353-62. Washington, DC, USA : IEEE Comput. Soc. Press, 1989, June 1989.

7. R. Gerth, R. Kupiter, and J. Segers. Interface refinement in reactive systems. In CONCUR '92 Third International Conference on Concurrency Theory, pages 77-93. Springer-Verlag, Aug 1992.

8. D. Greve. Symbolic simulation of the JEM1 microprocessor. In Formal Methods in Computer-Aided Design (FMCAD '98), volume 1522 of Lecture Notes in Computer Science, pages 321-333, 1998.

9. O. Grumberg and D. E. Long. Model checking and modular verification. ACM Transactions on Programming Languages and Systems, 16(3):843-871, May 1994.

10. R. Jhala and K. L. McMillan. Microarchitecture verification by compositional model checking. In A. Finkel G. Berry, H. Comon, editor, Computer Aided Verification: 13th International Conference, volume 2102 of Lecture Notes in Computer Science, pages 396-410. Springer-Verlag, 2001.

11. D. Kroning. Formal Verification of Pipelined Microprocessors. PhD thesis, Saarland University, 2001.

12. R. Kurki-Suonio. Component and interface refinement in closed-system specifications. In World Congress on Formal Methods (1), pages 134-154, 1999.

13. L. Lamport. What good is temporal logic? In R.E.A. Mason, editor, Information Processing, pages 657-668. Elsevier, 1983.

14. D. E. Long. Model Checking, Abstraction, and Compositional Verification. PhD thesis, Carnegie Mellon University, 1993.

15. K. L. McMillan. Verification of an implementation of Tomasulo's algorithm by compositional model checking. In A. J. Hu and M. Y. Vardi, editors, Computer Aided Verification (CAV'98), volume 1427 of LNCS. Springer-Verlag, June 1998.

16. K. L. McMillan. Getting started with SMV, March 1999.

17. K. L. McMillan. Parameterized verification of the flash cache coherence protocol by compositional model checking. In Correct Hardware Design and Verification Methods: 11th IFIP WG10.5 Advanced Research Working Conference, volume 2144 of Lecture Notes in Computer Science, pages 179-195. Springer-Verlag, 2001.

18. A. Rensink and R. Gorrieri. Vertical implementation. Information and Computation, 170:95-133, 2001. 